\title{
Soliton Solution and Conservation Law of Gear-Grimshaw Model for Shallow Water Waves
}

\author{
H. TRIKI ${ }^{a}$, A.H. KARA ${ }^{b}$, A.H. BhraWY ${ }^{c, d}$ AND A. Biswas ${ }^{e, c, *}$ \\ ${ }^{a}$ Radiation Physics Laboratory, Department of Physics, Faculty of Sciences, Badji Mokhtar University \\ P.O. Box 12, 23000 Annaba, Algeria \\ ${ }^{b}$ School of Mathematics, Centre for Differential Equations Continuum Mechanics and Applications \\ University of the Witwatersrand, Johannesburg, Wits 2050, South Africa \\ ${ }^{c}$ Department of Mathematics, Faculty of Science, King Abdulaziz University, Jeddah, Saudi Arabia \\ ${ }^{d}$ Department of Mathematics, Faculty of Science, Beni-Suef University, Beni-Suef, Egypt \\ ${ }^{e}$ Department of Mathematical Sciences, Delaware State University, Dover, DE 19901-2277, USA
}

(Received December 4, 2013)

This paper is going to obtain the soliton solution of the Gear-Grimshaw model that describes the dynamics of two-layered shallow water waves in oceans and rivers. The topological 1-soliton solution will be obtained by the ansatz method. There are several constraint conditions that will be taken care of. This will be followed by the model with power law nonlinearity. Subsequently, the conservation laws for this model will be derived by the aid of multiplier approach from the Lie symmetry analysis. Finally, the $F$-expansion method will extract a few more interesting solutions to the model.

DOI: 10.12693/APhysPolA.125.1099

PACS: 02.30.Ik, 02.30.Jr, 47.35.Fg

\section{Introduction}

The kinematics of shallow water waves can be described by several models [1-15]. Some of the commonly studied models are coupled Korteweg-de Vries $(\mathrm{KdV})$ equation, coupled Boussinesq equation, BonaChen equation [6] as well as the Gear-Grimshaw (GG) model [5] as well as several others. These model equations have been pretty exhaustively studied by several authors across the globe. The integrability aspect, exact solution, numerical simulations are all well studied.

This paper will shine some light on the GG model. It must be noted that GG equations have been addressed in the past both with regular as well as the power law nonlinear media. The soliton solutions, for each type of nonlinearity, has been obtained. The domain restrictions have been identified [5]. This paper is going to extract the shock wave solution for the GG model both with quadratic and power law nonlinear media. Subsequently, the multiplier approach, using Lie symmetry, will lead to the formulation of the conserved densities. The 1-soliton solution will then compute the conserved quantities for this equation.

\section{Mathematical analysis}

In this section the search is going to be for shock wave solution or topological 1-soliton solution to the GG model and its generalized form with power law nonlinearity. The study will therefore be split into the following two subsections.

*corresponding author; e-mail: biswas.anjan@gmail.com

\subsection{Quadratic nonlinearity}

The dimensionless form GG model that is going to be studied in this paper is given by $[5,12]$ :

$$
\begin{aligned}
& q_{t}+a q q_{x}+b q_{x x x}=\alpha_{1} r r_{x}+\beta_{1}(q r)_{x}+\gamma_{1} r_{x x x}, \\
& r_{t}+c r r_{x}+k r_{x x x}=\alpha_{2} q q_{x}+\beta_{2}(q r)_{x}+\gamma_{2} q_{x x x} .
\end{aligned}
$$

Here, the dependent variables are $q$ and $r$, while the independent variables are $x$ and $t$ which are the spatial and time variables, respectively. The coefficients $a, b, c, k$ and $\alpha_{j}, \beta_{j}, \gamma_{j}$ for $j=1,2$ are constants. In Ref. (1), the first term represents the evolution term, while $a$ and $b$, respectively, represent the coefficients of nonlinear and dispersion term. A similar situation with the coefficients of $c$ and $k$ for (2). Now $\alpha_{j}, \beta_{j}, \gamma_{j}$ for $j=1,2$ are all coupling terms. The search is going to be for 1-soliton solution to (1) and (2).

To start off, the hypothesis is given by [1]:

$$
q(x, t)=A_{1} \tanh ^{p_{1}} \tau
$$

and

$$
r(x, t)=A_{2} \tanh ^{p_{2}} \tau,
$$

where

$$
\tau=B(x-v t) .
$$
Eqs. (3)-(5), $A_{1}, A_{2}$, and $B$ are free parameter and $v$ is the velocity of the solitons. Also, $p_{1}$ and $p_{2}$ are unknown exponents that will be determined. Substituting these into (1) and (2) yields

$$
\begin{aligned}
& p_{1} v A_{1}\left(\tanh ^{p_{1}+1} \tau-\tanh ^{p_{1}-1} \tau\right) \\
& \quad+a p_{1} A_{1}^{2}\left(\tanh ^{2 p_{1}-1} \tau-\tanh ^{2 p_{1}+1} \tau\right) \\
& \quad+b p_{1} A_{1} B^{2}\left\{\left(p_{1}-1\right)\left(p_{1}-2\right) \tanh ^{p_{1}-3} \tau\right.
\end{aligned}
$$




$$
\begin{aligned}
& -\left[2 p_{1}^{2}+\left(p_{1}-1\right)\left(p_{1}-2\right)\right] \tanh ^{p_{1}-1} \tau \\
& +\left[2 p_{1}^{2}+\left(p_{1}+1\right)\left(p_{1}+2\right)\right] \tanh ^{p_{1}+1} \tau \\
& \left.-\left(p_{1}+1\right)\left(p_{1}+2\right) \tanh ^{p_{1}+3} \tau\right\} \\
& -\alpha_{1} p_{2} A_{2}^{2}\left(\tanh ^{2 p_{2}-1} \tau-\tanh ^{2 p_{2}+1} \tau\right) \\
& -\beta_{1} A_{1} A_{2}\left(p_{1}+p_{2}\right)\left(\tanh ^{p_{1}+p_{2}-1} \tau\right. \\
& \left.-\tanh ^{p_{1}+p_{2}+1} \tau\right)-\gamma_{1} p_{2} A_{2} B^{2}\left\{\left(p_{2}-1\right)\left(p_{2}-2\right)\right. \\
& \times \tanh ^{p_{2}-3} \tau-\left[2 p_{2}^{2}+\left(p_{2}-1\right)\left(p_{2}-2\right)\right] \tanh ^{p_{2}-1} \tau \\
& +\left[2 p_{2}^{2}+\left(p_{2}+1\right)\left(p_{2}+2\right)\right] \tanh ^{p_{2}+1} \tau \\
& \left.-\left(p_{2}+1\right)\left(p_{2}+2\right) \tanh ^{p_{2}+3} \tau\right\}=0
\end{aligned}
$$

and

$$
\begin{array}{rl}
p_{2} & v A_{2}\left(\tanh ^{p_{2}+1} \tau-\tanh ^{p_{2}-1} \tau\right) \\
& +c p_{2} A_{2}^{2}\left(\tanh ^{2 p_{2}-1} \tau-\tanh ^{2 p_{2}+1} \tau\right) \\
& +k p_{2} A_{2} B^{2}\left\{\left(p_{2}-1\right)\left(p_{2}-2\right) \tanh ^{p_{2}-3} \tau\right. \\
& -\left[2 p_{2}^{2}+\left(p_{2}-1\right)\left(p_{2}-2\right)\right] \tanh ^{p_{2}-1} \tau \\
& +\left[2 p_{2}^{2}+\left(p_{2}+1\right)\left(p_{2}+2\right)\right] \tanh ^{p_{2}+1} \tau \\
& \left.-\left(p_{2}+1\right)\left(p_{2}+2\right) \tanh ^{p_{2}+3} \tau\right\} \\
& -\alpha_{2} p_{1} A_{1}^{2}\left(\tanh ^{2 p_{1}-1} \tau-\tanh ^{2 p_{1}+1} \tau\right) \\
& -\beta_{2} A_{1} A_{2}\left(p_{1}+p_{2}\right)\left(\tanh ^{p_{1}+p_{2}-1} \tau\right. \\
- & \left.\tanh ^{p_{1}+p_{2}+1} \tau\right)-\gamma_{2} p_{1} A_{1} B^{2}\left\{\left(p_{1}-1\right)\left(p_{1}-2\right)\right. \\
& \times \tanh ^{p_{1}-3} \tau-\left[2 p_{1}^{2}+\left(p_{1}-1\right)\left(p_{1}-2\right)\right] \tanh ^{p_{1}-1} \tau \\
& +\left[2 p_{1}^{2}+\left(p_{1}+1\right)\left(p_{1}+2\right)\right] \tanh ^{p_{1}+1} \tau \\
& \left.-\left(p_{1}+1\right)\left(p_{1}+2\right) \tanh ^{p_{1}+3} \tau\right\}=0 .
\end{array}
$$

From (6), equating the exponents $p_{2}+3$ and $p_{1}+p_{2}+1$ gives

$$
p_{2}+3=p_{1}+p_{2}+1
$$

so that

$$
p_{1}=2 \text {. }
$$

The same result is yielded when the exponents $2 p_{1}+1$ and $p_{1}+3$ are equated. Now from (7), equating the exponents $2 p_{2}+1$ and $p_{2}+3$ gives

$$
2 p_{2}+1=p_{2}+3
$$

and therefore

$$
p_{2}=2 \text {. }
$$

It needs to be noted that the same value of $p$ is yielded when the exponents $p_{1}+3$ and $p_{1}+p_{2}+1$ are equated with each other.

Now from (6), the linearly independent functions are $\tanh \tau, \tanh ^{3} \tau$ and $\tanh ^{5} \tau$. Hence setting their respective coefficients to zero yields

$$
\begin{aligned}
& v=\frac{8 B^{2}\left(\gamma_{1} A_{2}-b A_{1}\right)}{A_{1}}, \\
& a A_{1}^{2}-\alpha_{1} A_{2}^{2}-2 \beta_{1} A_{1} A_{2} \\
& \quad+12 b A_{1} B^{2}-12 \gamma_{1} A_{2} B^{2}=0 .
\end{aligned}
$$

Again, from (7), setting the coefficients of the linearly independent functions $\tanh \tau, \tanh ^{3} \tau$ and $\tanh ^{5} \tau$ gives

$$
\begin{aligned}
& v=\frac{8 B^{2}\left(\gamma_{2} A_{1}-k A_{2}\right)}{A_{2}}, \\
& \alpha_{2} A_{1}^{2}-c A_{2}^{2}+2 \beta_{2} A_{1} A_{2} \\
& \quad-12 k A_{2} B^{2}+12 \gamma_{2} A_{1} B^{2}=0 .
\end{aligned}
$$

Now equating the two values of the velocity $v$ of the soliton from (12) and (14) gives

$$
(k-b) A_{2} A_{1}=\gamma_{2} A_{1}^{2}-\gamma_{1} A_{2}^{2},
$$

which serves as a constraint relation between the soliton amplitudes.

Thus, finally, the topological 1-soliton, or shock wave solutions to the considered Eqs. (1) and (2) are given by

$$
q(x, t)=A_{1} \tanh ^{2}(B(x-v t)),
$$

and

$$
r(x, t)=A_{2} \tanh ^{2}(B(x-v t)) .
$$

where the relation between the free parameters $A_{1}$ and $A_{2}$ is given by (16) while the velocity of the soliton is given by (12) or (14).

\subsection{Power law nonlinearity}

In this subsection, the GG model is going to be generalized to power law nonlinearity. Its dimensionless form is [5]:

$$
\begin{aligned}
& q_{t}+a q^{n} q_{x}+b q_{x x x}=\alpha_{1} r^{n} r_{x}+\beta_{1}\left(q^{n} r\right)_{x}+\gamma_{1} r_{x x x}, \\
& r_{t}+c r^{n} r_{x}+k r_{x x x}=\alpha_{2} q^{n} q_{x}+\beta_{2}\left(q r^{n}\right)_{x}+\gamma_{2} q_{x x x},
\end{aligned}
$$

where $n$ indicates the power law parameter. This model arises in a bounded domain with moving boundaries and non-homogeneous boundary conditions. It needs to be noted that Eqs. (19) and (20) condense to (1) and (2), respectively, for $n=1$. For solitary wave solutions, the starting assumptions are the same as in (3) and (4).

Substituting these hypothesis into (19) and (20) leads to

$$
\begin{array}{rl}
p_{1} & v A_{1}\left(\tanh ^{p_{1}+1} \tau-\tanh ^{p_{1}-1} \tau\right) \\
& +a p_{1} A_{1}^{n+1}\left(\tanh ^{p_{1}(n+1)-1} \tau-\tanh ^{p_{1}(n+1)+1} \tau\right) \\
& +b p_{1} A_{1} B^{2}\left\{\left(p_{1}-1\right)\left(p_{1}-2\right) \tanh ^{p_{1}-3} \tau\right. \\
& -\left[2 p_{1}^{2}+\left(p_{1}-1\right)\left(p_{1}-2\right)\right] \tanh ^{p_{1}-1} \tau \\
& +\left[2 p_{1}^{2}+\left(p_{1}+1\right)\left(p_{1}+2\right)\right] \tanh ^{p_{1}+1} \tau \\
& \left.-\left(p_{1}+1\right)\left(p_{1}+2\right) \tanh ^{p_{1}+3} \tau\right\}-\alpha_{1} p_{2} A_{2}^{n+1} \\
& \times\left(\tanh ^{p_{2}(n+1)-1} \tau-\tanh ^{p_{2}(n+1)+1} \tau\right) \\
& -\beta_{1} A_{1}^{n} A_{2}\left(n p_{1}+p_{2}\right)\left(\tanh ^{n p_{1}+p_{2}-1} \tau\right.
\end{array}
$$


$\left.-\tanh ^{n p_{1}+p_{2}+1} \tau\right)-\gamma_{1} p_{2} A_{2} B^{2}\left\{\left(p_{2}-1\right)\left(p_{2}-2\right)\right.$

$\times \tanh ^{p_{2}-3} \tau-\left[2 p_{2}^{2}+\left(p_{2}-1\right)\left(p_{2}-2\right)\right] \tanh ^{p_{2}-1} \tau$

$+\left[2 p_{2}^{2}+\left(p_{2}+1\right)\left(p_{2}+2\right)\right] \tanh ^{p_{2}+1} \tau$

$\left.-\left(p_{2}+1\right)\left(p_{2}+2\right) \tanh ^{p_{2}+3} \tau\right\}=0$

and

$$
\begin{array}{rl}
p_{2} & v A_{2}\left(\tanh ^{p_{2}+1} \tau-\tanh ^{p_{2}-1} \tau\right) \\
& +c p_{2} A_{2}^{n+1}\left(\tanh ^{p_{2}(n+1)-1} \tau-\tanh ^{p_{2}(n+1)+1} \tau\right) \\
& +k p_{2} A_{2} B^{2}\left\{\left(p_{2}-1\right)\left(p_{2}-2\right) \tanh ^{p_{2}-3} \tau\right. \\
& -\left[2 p_{2}^{2}+\left(p_{2}-1\right)\left(p_{2}-2\right)\right] \tanh ^{p_{2}-1} \tau \\
& +\left[2 p_{2}^{2}+\left(p_{2}+1\right)\left(p_{2}+2\right)\right] \tanh ^{p_{2}+1} \tau \\
& \left.-\left(p_{2}+1\right)\left(p_{2}+2\right) \tanh ^{p_{2}+3} \tau\right\}-\alpha_{2} p_{1} A_{1}^{n+1} \\
& \times\left(\tanh ^{p_{1}(n+1)-1} \tau-\tanh ^{p_{1}(n+1)+1} \tau\right) \\
& -\beta_{2} A_{1} A_{2}^{n}\left(p_{1}+n p_{2}\right)\left(\tanh ^{p_{1}+n p_{2}-1} \tau\right. \\
& \left.-\tanh ^{p_{1}+n p_{2}+1} \tau\right)-\gamma_{2} p_{1} A_{1} B^{2}\left\{\left(p_{1}-1\right)\left(p_{1}-2\right)\right. \\
& \times \tanh ^{p_{1}-3} \tau-\left[2 p_{1}^{2}+\left(p_{1}-1\right)\left(p_{1}-2\right)\right] \tanh ^{p_{1}-1} \tau \\
& +\left[2 p_{1}^{2}+\left(p_{1}+1\right)\left(p_{1}+2\right)\right] \tanh ^{p_{1}+1} \tau \\
& \left.-\left(p_{1}+1\right)\left(p_{1}+2\right) \tanh ^{p_{1}+3} \tau\right\}=0,
\end{array}
$$

respectively.

Now from (21), equating the exponents $p_{2}+3$ and $n p_{1}+p_{2}+1$ gives

$$
p_{2}+3=n p_{1}+p_{2}+1
$$

so that

$$
p_{1}=2 / n \text {. }
$$

The same result is yielded when the exponents pairs $p_{1}(n+1)+1$ and $p_{1}+3, p_{1}(n+1)-1$ and $p_{1}+1$ are equated.

Similarly, from (22), equating the exponents $p_{2}(n+$ 1) +1 and $p_{2}+3$ gives

$$
p_{2}(n+1)+1=p_{2}+3
$$

and therefore

$$
p_{2}=2 / n \text {. }
$$

It needs to be observed that the same value of $p$ is yielded when the exponents $p_{1}+3$ and $p_{1}+n p_{2}+1$ are equated with each other.

Then, setting the coefficient of the stand-alone linearly independent function $\tanh ^{p-3} \tau$ in (21) to zero yields

$$
\begin{aligned}
p_{1} & =p_{2}=1 \\
\text { or } & \\
p_{1} & =p_{2}=2 .
\end{aligned}
$$

This leads to

$$
n=1 \text { or } n=2 \text {. }
$$

Upon setting $n=1$, the same results as those for Eqs. (1) and (2), namely quadratic nonlinearity, are obtained. Therefore, this study will focus on the case $n=2$. For such situation, the linearly independent functions in (21) are $\tanh ^{0} \tau, \tanh ^{2} \tau$ and $\tanh ^{4} \tau$. Hence setting their respective coefficients to zero yields

$$
\begin{aligned}
& v=\frac{2 B^{2}\left(\gamma_{1} A_{2}-b A_{1}\right)}{A_{1}}, \\
& a A_{1}^{3}+6 b A_{1} B^{2}-\alpha_{1} A_{2}^{3}-3 \beta_{1} A_{1}^{2} A_{2}-6 \gamma_{1} A_{2} B^{2}=0 .
\end{aligned}
$$

Considering the case $n=2$ (namely and $p_{1}=p_{2}=1$ ) in $(22)$ and setting the coefficients of the linearly independent functions $\tanh ^{0} \tau, \tanh ^{2} \tau$ and $\tanh ^{4} \tau$ to zero gives

$$
\begin{aligned}
& v=\frac{2 B^{2}\left(\gamma_{2} A_{1}-k A_{2}\right)}{A_{2}}, \\
& c p_{2} A_{2}^{3}+6 k p_{2} A_{2} B^{2}-\alpha_{2} p_{1} A_{1}^{3}-3 \beta_{2} A_{1} A_{2}^{2} \\
& \quad-6 \gamma_{2} p_{1} A_{1} B^{2} .
\end{aligned}
$$

Now equating the two values of the velocity $v$ of the soliton from (30) and (32) gives

$$
(k-b) A_{1} A_{2}=\gamma_{2} A_{1}^{2}-\gamma_{1} A_{2}^{2},
$$

which serves as a constraint relation between the soliton free parameters $A_{1}$ and $A_{2}$.

This shows that for the GG model with power law nonlinearity (19) and (20), topological solitons exist only for $n=1$ and 2. This is a very important observation that is being made for the first time in this paper.

Thus, finally, the topological 1-soliton solutions to (19) and (20) are given by

$$
q(x, t)=A_{1} \tanh (B(x-v t)),
$$

and

$$
r(x, t)=A_{2} \tanh (B(x-v t)),
$$

where the relation between the free parameters $A_{1}$ and $A_{2}$ is given by (34) while the velocity of the soliton is given by (30) or (32).

\section{Conservation law}

In order to determine conserved densities and fluxes, we resort to the invariance and multiplier approach based on the well known result that the Euler-Lagrange operator annihilates a total divergence (see [7]). Firstly, if $\left(T^{t}, T^{x}\right)$ is a conserved vector corresponding to a conservation law, then

$$
D_{t} T^{t}+D_{x} T^{x}=0
$$

along the solutions of the system of differential equations $G^{1}\left(x, t, q, r, q_{(1)}, r_{(1)}, \ldots\right)=0$ and $G^{2}\left(x, t, q, r, q_{(1)}, r_{(1)}, \ldots\right)=0$. If there exists a vector $Q=\left(Q^{1}, q^{2}\right)$, say, so that

$$
Q^{1}\left(G^{1}\right)+Q^{2}\left(G^{2}\right)=D_{t} T^{t}+D_{x} T^{x},
$$

then

$$
E_{(q, r)}\left[D_{t} T^{t}+D_{x} T^{x}\right]=0,
$$

where $E_{(q, r)}$ is the respective Euler operator. In each case, $T^{t}$ is the conserved density. 
The lengthy calculations for the above system lead to the only multiplier $\left(Q^{1}, Q^{2}\right)=(1,1)$ and corresponding conserved vector is given by

$$
\begin{aligned}
T^{x} & =\frac{1}{n+1}\left\{\left(a-\alpha_{2}\right) q^{1+n}-(1+n) \beta_{1} q^{n} r\right. \\
& -(1+n) \beta_{2} q r^{n}+\left(c-\alpha_{1}\right) r^{1+n}+(1+n) \\
& \left.\times\left[\left(b-\gamma_{2}\right) q_{x x}+\left(d-\gamma_{1}\right) r_{x x}\right]\right\}, \\
T^{t} & =\int_{0}^{1}\left(a \lambda^{n} q^{1+n}-\lambda^{n} \alpha_{2} q^{1+n}-\lambda^{n} \beta_{1} q^{n} r\right. \\
& -n \lambda^{n} \beta_{1} q^{n} r-\lambda^{n} \beta_{2} q r^{n}-n \lambda^{n} \beta_{2} q r^{n}+c \lambda^{n} r^{1+n} \\
- & \left.\lambda^{n} \alpha_{1} r^{1+n}+b q_{x x}-\gamma_{2} q_{x x}+d r_{x x}-\gamma_{1} r_{x x}\right) \mathrm{d} \lambda \\
& =\frac{1}{n+1}\left(a q^{1+n}-\alpha_{2} q^{1+n}-\beta_{1} q^{n} r-n \beta_{1} q^{n} r\right. \\
- & \left.\beta_{2} q r^{n}-n \beta_{2} q r^{n}+c r^{1+n}-\alpha_{1} r^{1+n}\right) \\
+ & \left(b q_{x x}-\gamma_{2} q_{x x}+d r_{x x}-\gamma_{1} r_{x x}\right) .
\end{aligned}
$$

In order to obtain the conserved quantity from the conserved density, the 1-soliton solution to the GG model with power law nonlinearity is given by [5]:

and

$$
q(x, t)=A_{1} \operatorname{sech}^{\frac{2}{n}}(B(x-v t))
$$

$$
r(x, t)=A_{2} \operatorname{sech}^{\frac{2}{n}}(B(x-v t)),
$$

where the velocity $(v)$ of the solitons is given by

$$
v=\left[4 B^{2}\left(b A_{1}-\gamma_{1} A_{2}\right)\right] /\left(n^{2} A_{1}\right)
$$

Or

$$
v=\left[4 B^{2}\left(k A_{2}-\gamma_{2} A_{1}\right)\right] /\left(n^{2} A_{2}\right) .
$$

Upon equating the two values of the velocity $v$ of the soliton from (39) and (40) leads to the relation between the amplitudes of the solitons as

$$
(b-k) A_{1} A_{2}=\gamma_{1} A_{2}^{2}-\gamma_{2} A_{1}^{2} .
$$

These soliton solutions will be used to compute the conserved quantity from its density.

The conserved quantity is now computed as

$$
\begin{aligned}
I & =\int_{-\infty}^{\infty} T^{t} \mathrm{~d} x=\frac{1}{n+1} \int_{-\infty}^{\infty}\left\{\left(a-\alpha_{2}\right) q^{n+1}-(n+1)\right. \\
& \times\left[\beta_{1} q^{n} r+\beta_{2} q r^{n}\right]+\left(c-\alpha_{1}\right) r^{n+1}+\left(b-\gamma_{2}\right) q_{x x} \\
& \left.+\left(d-\gamma_{1}\right) r_{x x}\right\} \mathrm{d} x=\frac{2}{(n+1)(n+2) B} \\
& \times\left[\left(a-\alpha_{2}\right) A_{1}^{n+1}-(n+1)\left(\beta_{1} A_{1}^{n} A_{2}+\beta_{2} A_{1} A_{2}^{n}\right)\right. \\
& \left.+\left(c-\alpha_{1}\right) A_{2}^{n+1}\right]\left(\Gamma\left(\frac{1}{2}\right) \Gamma\left(\frac{1}{n}\right)\right) /\left(\Gamma\left(\frac{1}{n}+\frac{1}{2}\right)\right) .
\end{aligned}
$$

Upon setting $n=1$, the conservation law of the special case of quadratic nonlinearity is recovered.

\section{4. $\boldsymbol{F}$-expansion method}

In this section, we introduce a simple description of the $F$-expansion (FE) method (see for instance [1-3]), for a given partial differential equation

$$
G\left(u, u_{x}, u_{y}, u_{z}, u_{x y}, \ldots\right)=0 .
$$

We like to know whether traveling waves (or stationary waves) are solutions of Eq. (43). The first step is to unite the independent variables $x, y$ and $t$ into one particular variable through the new variable

$$
\zeta=x+y-\nu t, \quad u(x, y, t)=U(\zeta),
$$

where $\nu$ is wave speed, and reduce Eq. (43) to an ordinary differential equation (ODE)

$$
G\left(U, U^{\prime}, U^{\prime \prime}, U^{\prime \prime \prime}, \ldots\right)=0 \text {. }
$$

Our main goal is to derive exact or at least approximate solutions, if possible, for this ODE. For this purpose, the solutions $U$ can be proposed as a finite power series in the form,

$$
u(x, y, t)=U(\zeta)=\sum_{i=0}^{N} a_{i} F^{i}
$$

where

$$
F^{\prime}=\sqrt{A+B F^{2}+C F^{4}}
$$

we take $F$ as the Jacobi elliptic function (sn, cn, dn, ...) since $F$ here stands for each of the Jacobi elliptic functions, for example if we take $F(\zeta)=\operatorname{sn}(\zeta)$, we find

$$
\begin{aligned}
F^{\prime} & =\operatorname{cn}(\zeta) \operatorname{dn}(\zeta)=\sqrt{1-\mathrm{sn}^{2}(\zeta)} \sqrt{1-m^{2} \operatorname{sn}^{2}(\zeta)} \\
& =\sqrt{1-\left(1+m^{2}\right) \operatorname{sn}^{2}(\zeta)+m^{2} \operatorname{sn}^{4}(\zeta)},
\end{aligned}
$$

moreover, any derivative of the Jacobi elliptic function (triangle and hyperbolic function) may be put in the form of Eq. (46) which meets the Hamilton invariable. Therefore, $F^{\prime}$ in Eq. (7), is taken in a general form. The highest degree of $\frac{\mathrm{d}^{p} U}{\mathrm{~d} \zeta^{p}}$ is taken as

$$
\begin{gathered}
\mathrm{O}\left(\frac{\mathrm{d}^{p} U}{\mathrm{~d} \zeta^{p}}\right)=N+p, \quad p=1,2,3, \ldots, \\
\mathrm{O}\left(U^{q} \frac{\mathrm{d}^{p} U}{\mathrm{~d} \zeta^{p}}\right)=(q+1) N+p, \\
q=0,1,2, \ldots, \quad p=1,2,3, \ldots
\end{gathered}
$$

where $A, B$, and $C$ are constants, and $N$ in Eq. (44) is a positive integer that can be determined by balancing the nonlinear term(s) and the highest order derivatives. Normally $N$ is a positive integer, so that an analytic solution in closed form may be obtained. Substituting Eqs. (43)-(46) into Eq. (44) and comparing the coefficients of each power of $F(\zeta)$ in both sides, we get an over-determined system of nonlinear algebraic equations with respect to $\nu, a_{0}, a_{1}, \ldots$ Solving of the over-determined system of nonlinear algebraic equations are performed by use of Mathematica. The relations between values of $A$, $B, C$ and corresponding Jacobian elliptic function (JEF) solution $F(\zeta)$ of Eq. (45) are given in Table. Substitute the values of $A, B, C$ and the corresponding JEF solution $F(\zeta)$ chosen from Table into the general form of solution, then an ideal periodic wave solution expressed by JEF can be obtained.

$\operatorname{sn}(\zeta), \operatorname{cn}(\zeta)$ and $\operatorname{dn}(\zeta)$ are the JE sine function, JE cosine function, and the JEF of the third kind, respectively, and

$$
\operatorname{cn}^{2}(\zeta)=1-\operatorname{sn}^{2}(\zeta), \quad \operatorname{dn}^{2}(\zeta)=1-m^{2} \operatorname{sn}^{2}(\zeta),
$$

with the modulus $m(0<m<1)$.

When $m \longrightarrow 1$, the Jacobi functions degenerate to the hyperbolic functions, i.e.,

$\operatorname{sn} \zeta \longrightarrow \tanh \zeta, \quad \operatorname{cn} \zeta \longrightarrow \operatorname{sech} \zeta, \operatorname{dn} \zeta \longrightarrow \operatorname{sech} \zeta$ 
TABLE

Relation between values of $(A, B, C)$ and corresponding $F$.

\begin{tabular}{c|c|c|c}
\hline \hline$A$ & $B$ & $C$ & $F(\zeta)$ \\
\hline 1 & $-1-m^{2}$ & $m^{2}$ & $\operatorname{sn}(\zeta)$ or \\
$\operatorname{cd}(\zeta)=\frac{\operatorname{cn}(\zeta)}{\operatorname{dn}(\zeta)}$ \\
$1-m^{2}$ & $2 m^{2}-1$ & $-m^{2}$ & $\operatorname{cn}(\zeta)$ \\
$m^{2}-1$ & $2-m^{2}$ & -1 & $\operatorname{dn}(\zeta)$ \\
$m^{2}$ & $-1-m^{2}$ & 1 & $\operatorname{ns}(\zeta)=\frac{1}{\operatorname{sn}(\zeta)}$ \\
$-m^{2}$ & $2 m^{2}-1$ & $1-m^{2}$ & $\operatorname{or~} \operatorname{dc}(\zeta)=\frac{\mathrm{dn}(\zeta)}{\operatorname{cn}(\zeta)}$ \\
-1 & $2-m^{2}$ & $m^{2}-1$ & $\operatorname{nc}(\zeta)=\frac{1}{\operatorname{cn}(\zeta)}$ \\
1 & $2-m^{2}$ & $1-m^{2}$ & $\operatorname{nd}(\zeta)=\frac{1}{\operatorname{dn}(\zeta)}$ \\
1 & $2 m^{2}-1$ & $-m^{2}\left(-1-m^{2}\right)$ & $\operatorname{sd}(\zeta)=\frac{\operatorname{sn}(\zeta)}{\operatorname{cn}(\zeta)}$ \\
$1-m^{2}(\zeta)$ & $2-m^{2}$ & 1 & $\operatorname{cs}(\zeta)=\frac{\operatorname{cn}(\zeta)}{\operatorname{sn}(\zeta)}$ \\
$-m^{2}\left(1-m^{2}\right)$ & $2 m^{2}-1$ & 1 & $\operatorname{ds}(\zeta)=\frac{\operatorname{dn}(\zeta)}{\operatorname{sn}(\zeta)}$ \\
$\frac{1}{4}$ & $\frac{1-2 m^{2}}{2}$ & $\frac{1}{4}$ & $\operatorname{ns}(\zeta)+\operatorname{cs}(\zeta)$ \\
$\frac{1-m^{2}}{4}$ & $\frac{1+m^{2}}{2}$ & $\frac{1-m^{2}}{2}$ & $\operatorname{nc}(\zeta)+\operatorname{sc}(\zeta)$ \\
$\frac{1}{4}$ & $\frac{m^{2}-2}{2}$ & $\frac{m^{2}}{4}$ & $\operatorname{ns}(\zeta)+\operatorname{ds}(\zeta)$ \\
$\frac{m^{2}}{4}$ & $\frac{m^{2}-2}{2}$ & $\frac{m^{2}}{4}$ & $\operatorname{sn}(\zeta)+\operatorname{ics}(\zeta)$
\end{tabular}

when $m \longrightarrow 0$, the Jacobi functions degenerate to the triangular functions, i.e.,

$\operatorname{sn} \zeta \longrightarrow \sin \zeta, \quad \operatorname{cn} \zeta \longrightarrow \cos \zeta \quad$ and $\quad \mathrm{dn} \longrightarrow 1$.

This analysis will be applied to the GG model that will be split into the following couple of subsections that depend on the type of nonlinearity.

\subsection{Quadratic nonlinearity}

The dimensionless form GG model is

$$
\begin{aligned}
& q_{t}+a q q_{x}+b q_{x x x}=\alpha_{1} r r_{x}+\beta_{1}(q r)_{x}+\gamma_{1} r_{x x x}, \\
& r_{t}+c r r_{x}+k r_{x x x}=\alpha_{2} q q_{x}+\beta_{2}(q r)_{x}+\gamma_{2} q_{x x x},
\end{aligned}
$$

as described in the introductory section, we perform a travelling wave reduction,

$$
u(x, t)=U(\zeta), \quad v(x, t)=V(\zeta), \quad \zeta=x+\nu t,
$$

that converted (50) into a system of ODEs,

$$
\begin{aligned}
& \nu Q^{\prime}+a Q Q^{\prime}+b Q^{\prime \prime \prime}=\alpha_{1} R R^{\prime}+\beta_{1}(Q R)^{\prime}+\gamma_{1} R^{\prime \prime \prime}, \\
& \nu R^{\prime}+c R R^{\prime}+k R^{\prime \prime \prime}=\alpha_{2} Q Q^{\prime}+\beta_{2}(Q R)^{\prime}+\gamma_{2} Q^{\prime \prime \prime},(52
\end{aligned}
$$

if we integrate the system (52) once, upon setting the constant of integration to zero, we obtain

$$
\begin{aligned}
& 2 \nu Q+a Q^{2}+2 b Q^{\prime \prime}=\alpha_{1} R^{2}+2 \beta_{1} Q R+2 \gamma_{1} R^{\prime \prime}, \\
& 2 \nu R+c R^{2}+2 k R^{\prime \prime}=\alpha_{2} Q^{2}+2 \beta_{2} Q R+2 \gamma_{2} Q^{\prime \prime} .
\end{aligned}
$$

Balancing the term $Q^{\prime \prime}$ with the term $Q R$, and $R^{\prime \prime}$ with the term $Q R$ implies to $N=2$ and $M=2$. Accordingly, the solution takes on the form

$$
Q(\zeta)=\sum_{i=0}^{2} a_{i}(F)^{i}, \quad R(\zeta)=\sum_{i=0}^{2} b_{i}(F)^{i} .
$$

Substituting Eq. (54) into Eq. (53) and comparing the coefficients of each power of $F$ in both sides, getting an over-determined system of nonlinear algebraic equations with respect to $\nu, \mu, \lambda$ and $a_{i}, i=0,2$, we obtain

$$
a_{0}=a_{1}=b_{0}=b_{1}=A=0,
$$

$$
a_{2}=
$$$$
-\frac{48 B C \nu \gamma_{1}^{2}}{16 b^{2} B^{2} \alpha_{1}+8 b B \nu \alpha_{1}+\nu^{2} \alpha_{1}+32 b B^{2} \beta_{1} \gamma_{1}+8 B \nu \beta_{1} \gamma_{1}-16 a B^{2} \gamma_{1}^{2}},
$$$$
b_{2}=
$$$$
-\frac{12 C \nu(4 b B+\nu) \gamma_{1}}{16 b^{2} B^{2} \alpha_{1}+8 b B \nu \alpha_{1}+\nu^{2} \alpha_{1}+32 b B^{2} \beta_{1} \gamma_{1}+8 B \nu \beta_{1} \gamma_{1}-16 a B^{2} \gamma_{1}^{2}},
$$$$
k=\frac{-4 b B \nu-\nu^{2}+16 B^{2} \gamma_{1} \gamma_{2}}{4 B(4 b B+\nu)}
$$

where the relation between the free parameters is

$$
\begin{aligned}
& C \nu\left[(4 b B+\nu)^{3} \alpha_{1}+4 B \gamma_{1}(4 b B+\nu)^{2}\left(c+2 \beta_{1}\right)\right. \\
& \left.\quad-4 B(4 b B+\nu)\left(a+2 \beta_{2}\right) \gamma_{1}-16 B^{2} \alpha_{2} \gamma_{1}^{2}\right]=0,
\end{aligned}
$$

the solutions of Eq, (50) may be given by

$$
\begin{aligned}
& q_{1}(x, t)=\frac{4 \csc (x+\nu t)^{2} b_{2} \gamma_{1}}{-4 b+\nu}, \\
& r_{1}(x, t)=-\frac{12 \nu(-4 b+\nu) \csc (x+\nu t)^{2} \gamma_{1}}{16 b^{2} \alpha_{1}-8 b \nu \alpha_{1}+\nu^{2} \alpha_{1}+32 b \beta_{1} \gamma_{1}-8 \nu \beta_{1} \gamma_{1}-16 a \gamma_{1}^{2}}, \\
& q_{2}(x, t)=-\frac{4 \sec (x+\nu t)^{2} b_{2} \gamma_{1}}{-4 b+\nu}, \\
& r_{2}(x, t)=-\frac{12 \nu(-4 b+\nu) \sec (x+\nu t)^{2} \gamma_{1}}{16 b^{2} \alpha_{1}-8 b \nu \alpha_{1}+\nu^{2} \alpha_{1}+32 b \beta_{1} \gamma_{1}-8 \nu \beta_{1} \gamma_{1}-16 a \gamma_{1}^{2}}, \\
& q_{3}(x, t)=\frac{4 \operatorname{sech}(x+\nu t)^{2} b_{2} \gamma_{1}}{4 b+\nu}, \\
& r_{3}(x, t)=\frac{12 \nu(4 b+\nu) \operatorname{sech}(x+\nu t)^{2} \gamma_{1}}{16 b^{2} \alpha_{1}+8 b \nu \alpha_{1}+\nu^{2} \alpha_{1}+32 b \beta_{1} \gamma_{1}+8 \nu \beta_{1} \gamma_{1}-16 a \gamma_{1}^{2}}, \\
& q_{4}(x, t)=\frac{4 \operatorname{csch}(x+\nu t)^{2} b_{2} \gamma_{1}}{4 b+\nu}, \\
& r_{4}(x, t)=-\frac{12 \nu(4 b+\nu) \operatorname{csch}(x+\nu t)^{2} \gamma_{1}}{16 b^{2} \alpha_{1}+8 b \nu \alpha_{1}+\nu^{2} \alpha_{1}+32 b \beta_{1} \gamma_{1}+8 \nu \beta_{1} \gamma_{1}-16 a \gamma_{1}^{2}} .
\end{aligned}
$$

\subsection{Power law nonlinearity}

The dimensionless form GG model is

$$
\begin{aligned}
& q_{t}+a q^{2} q_{x}+b q_{x x x}=\alpha_{1} r^{2} r_{x}+\beta_{1}\left(q^{2} r\right)_{x}+\gamma_{1} r_{x x x}, \\
& r_{t}+c r^{2} r_{x}+k r_{x x x}=\alpha_{2} q^{2} q_{x}+\beta_{2}\left(q r^{2}\right)_{x}+\gamma_{2} q_{x x x}
\end{aligned}
$$

as described in the introductory section, we perform a travelling wave reduction,

$$
u(x, t)=U(\zeta), \quad v(x, t)=V(\zeta), \quad \zeta=x+\nu t,
$$

that converted (55) into a system of ODEs,

$$
\begin{aligned}
& \nu Q^{\prime}+a Q^{2} Q^{\prime}+b Q^{\prime \prime \prime}=\alpha_{1} R^{2} R^{\prime}+\beta_{1}\left(Q^{2} R\right)^{\prime}+\gamma_{1} R^{\prime \prime \prime}, \\
& \nu R^{\prime}+c R^{2} R^{\prime}+k R^{\prime \prime \prime}=\alpha_{2} Q^{2} Q^{\prime}+\beta_{2}\left(Q R^{2}\right)^{\prime}+\gamma_{2} Q^{\prime \prime \prime},
\end{aligned}
$$

if we integrate the system (57) once, upon setting the constant of integration to zero, we obtain

$$
\begin{aligned}
& 3 \nu Q+a Q^{3}+3 b Q^{\prime \prime}=\alpha_{1} R^{3}+3 \beta_{1} Q^{2} R+3 \gamma_{1} R^{\prime \prime}, \\
& 3 \nu R+c R^{3}+3 k R^{\prime \prime}=\alpha_{2} Q^{3}+3 \beta_{2} Q R^{2}+3 \gamma_{2} Q^{\prime \prime} .
\end{aligned}
$$

Balancing the term $Q^{\prime \prime}$ with the term $Q^{2} R$, and $R^{\prime \prime}$ with the term $Q R^{2}$ implies to $N=1$ and $M=1$. Accordingly, the solution takes on the form

$$
Q(\zeta)=\sum_{i=0}^{1} a_{i}(F)^{i}, \quad R(\zeta)=\sum_{i=0}^{1} b_{i}(F)^{i} .
$$


Substituting Eq. (59) into Eq. (58) and comparing the coefficients of each power of $F$ in both sides, getting an over-determined system of nonlinear algebraic equations with respect to $\nu, \mu, \lambda$ and $a_{i}, i=0,1$. We obtain

$$
\begin{aligned}
& a_{0}=b_{0}=0, \\
& a_{1}=-\frac{\mathrm{i} \sqrt{6} B \sqrt{C} \sqrt{\nu} \gamma_{1}^{3 / 2}}{\sqrt{b^{3} B^{3} \alpha_{1}+3 b^{2} B^{2} \nu \alpha_{1}+3 b B \nu^{2} \alpha_{1}+\nu^{3} \alpha_{1}+3 b B^{3} \beta_{1} \gamma_{1}^{2}+3 B^{2} \nu \beta_{1} \gamma_{1}^{2}-a B^{3} \gamma_{1}^{3}}}, \\
& b_{1}=-\frac{\mathrm{i} \sqrt{6} \sqrt{C} \sqrt{\nu}(b B+\nu) \sqrt{\gamma_{1}}}{\sqrt{b^{3} B^{3} \alpha_{1}+3 b^{2} B^{2} \nu \alpha_{1}+3 b B \nu^{2} \alpha_{1}+\nu^{3} \alpha_{1}+3 b B^{3} \beta_{1} \gamma_{1}^{2}+3 B^{2} \nu \beta_{1} \gamma_{1}^{2}-a B^{3} \gamma_{1}^{3}}}, \\
& k=\frac{-b B \nu-\nu^{2}+B^{2} \gamma_{1} \gamma_{2}}{B(b B+\nu)},
\end{aligned}
$$

where the relation between the free parameters is

$$
-\frac{\nu(b B+\nu)}{B}+\frac{\nu \gamma_{1}\left[-c(b B+\nu)^{3}+3 B(b B+\nu)^{2} \beta_{2} \gamma_{1}+B^{3} \alpha_{2} \gamma_{1}^{3}\right]}{(b B+\nu)^{3} \alpha_{1}+B^{2} \gamma_{1}^{2}\left[3(b B+\nu) \beta_{1}-a B \gamma_{1}\right]}=0
$$

the solutions of Eq. (55) may be given by

$$
\begin{aligned}
& q_{1}(x, t)=\frac{\sqrt{6} \sqrt{m^{2}}\left(-1-m^{2}\right) \sqrt{\nu} \operatorname{sn}(x+\nu t) \gamma_{1}^{3 / 2}}{\sqrt{\left(b+b m^{2}-\nu\right)^{3} \alpha_{1}-\left(1+m^{2}\right)^{2} \gamma_{1}^{2}\left[-3\left(b+b m^{2}-\nu\right) \beta_{1}+a\left(1+m^{2}\right) \gamma_{1}\right]}}, \\
& r_{1}(x, t)=\frac{\sqrt{6} \sqrt{m^{2}} \sqrt{\nu}\left[b\left(-1-m^{2}\right)+\nu\right] \operatorname{sn}(x+\nu t) \sqrt{\gamma_{1}}}{\sqrt{\left(b+b m^{2}-\nu\right)^{3} \alpha_{1}-\left(1+m^{2}\right)^{2} \gamma_{1}^{2}\left[-3\left(b+b m^{2}-\nu\right) \beta_{1}+a\left(1+m^{2}\right) \gamma_{1}\right]}}, \\
& q_{2}(x, t)=-\frac{\sqrt{6}\left(-1-m^{2}\right) \sqrt{\nu} \operatorname{ns}(x+\nu t) \gamma_{1}^{3 / 2}}{\sqrt{\left(b+b m^{2}-\nu\right)^{3} \alpha_{1}-\left(1+m^{2}\right)^{2} \gamma_{1}^{2}\left[-3\left(b+b m^{2}-\nu\right) \beta_{1}+a\left(1+m^{2}\right) \gamma_{1}\right]}}, \\
& r_{2}(x, t)=-\frac{\sqrt{6} \sqrt{\nu}\left[b\left(-1-m^{2}\right)+\nu\right] \operatorname{ns}(x+\nu t) \sqrt{\gamma_{1}}}{\sqrt{\left(b+b m^{2}-\nu\right)^{3} \alpha_{1}-\left(1+m^{2}\right)^{2} \gamma_{1}^{2}\left[-3\left(b+b m^{2}-\nu\right) \beta_{1}+a\left(1+m^{2}\right) \gamma_{1}\right]}}, \\
& q_{3}(x, t)=\frac{\sqrt{6}\left(-1-m^{2}\right) \sqrt{\nu} \mathrm{dc}(x+\nu t) \gamma_{1}^{3 / 2}}{\sqrt{\left(b+b m^{2}-\nu\right)^{3} \alpha_{1}-\left(1+m^{2}\right)^{2} \gamma_{1}^{2}\left[-3\left(b+b m^{2}-\nu\right) \beta_{1}+a\left(1+m^{2}\right) \gamma_{1}\right]}}, \\
& r_{3}(x, t)=-\frac{\sqrt{6} \sqrt{\nu}\left[b\left(-1-m^{2}\right)+\nu\right] \mathrm{dc}(x+\nu t) \sqrt{\gamma_{1}}}{\sqrt{\left(b+b m^{2}-\nu\right)^{3} \alpha_{1}-\left(1+m^{2}\right)^{2} \gamma_{1}^{2}\left[-3\left(b+b m^{2}-\nu\right) \beta_{1}+a\left(1+m^{2}\right) \gamma_{1}\right]}}, \\
& q_{4}(x, t)=-\frac{\sqrt{6} \sqrt{m^{2}}\left(-1-m^{2}\right) \sqrt{\nu} \operatorname{cd}(x+\nu t) \gamma_{1}^{3 / 2}}{\sqrt{\left(b+b m^{2}-\nu\right)^{3} \alpha_{1}-\left(1+m^{2}\right)^{2} \gamma_{1}^{2}\left[-3\left(b+b m^{2}-\nu\right) \beta_{1}+a\left(1+m^{2}\right) \gamma_{1}\right]}}, \\
& r_{4}(x, t)=\frac{\sqrt{6} \sqrt{m^{2}} \sqrt{\nu}\left[b\left(-1-m^{2}\right)+\nu\right] \operatorname{cd}(x+\nu t) \sqrt{\gamma_{1}}}{\sqrt{\left(b+b m^{2}-\nu\right)^{3} \alpha_{1}-\left(1+m^{2}\right)^{2} \gamma_{1}^{2}\left[-3\left(b+b m^{2}-\nu\right) \beta_{1}+a\left(1+m^{2}\right) \gamma_{1}\right]}}, \\
& q_{5}(x, t)=-\frac{\mathrm{i} \sqrt{6} \sqrt{-m^{2}}\left(-1+2 m^{2}\right) \sqrt{\nu} \operatorname{cn}(x+\nu t) \gamma_{1}^{3 / 2}}{\sqrt{\left[b\left(-1+2 m^{2}\right)+\nu\right]^{3} \alpha_{1}+\left(1-2 m^{2}\right)^{2} \gamma_{1}^{2}\left[3\left(-b+2 b m^{2}+\nu\right) \beta_{1}+a\left(1-2 m^{2}\right) \gamma_{1}\right]}}, \\
& r_{5}(x, t)=-\frac{\mathrm{i} \sqrt{6} \sqrt{-m^{2}} \sqrt{\nu}\left[b\left(-1+2 m^{2}\right)+\nu\right] \operatorname{cn}(x+\nu t) \sqrt{\gamma_{1}}}{\sqrt{\left[b\left(-1+2 m^{2}\right)+\nu\right]^{3} \alpha_{1}+\left(1-2 m^{2}\right)^{2} \gamma_{1}^{2}\left[3\left(-b+2 b m^{2}+\nu\right) \beta_{1}+a\left(1-2 m^{2}\right) \gamma_{1}\right]}},
\end{aligned}
$$




$$
\begin{aligned}
& q_{6}(x, t)=-\frac{\sqrt{6} \sqrt{-1}\left(2-m^{2}\right) \sqrt{\nu} \operatorname{dn}(x+\nu t) \gamma_{1}^{3 / 2}}{\sqrt{\left[b\left(-2+m^{2}\right)-\nu\right]^{3} \alpha_{1}-\left(-2+m^{2}\right)^{2} \gamma_{1}^{2}\left[3\left(2 b-b m^{2}+\nu\right) \beta_{1}+a\left(-2+m^{2}\right) \gamma_{1}\right]}}, \\
& r_{6}(x, t)=-\frac{\sqrt{6} \sqrt{-1} \sqrt{\nu}\left[b\left(2-m^{2}\right)+\nu\right] \operatorname{dn}(x+\nu t) \sqrt{\gamma_{1}}}{\sqrt{\left[b\left(-2+m^{2}\right)-\nu\right]^{3} \alpha_{1}-\left(-2+m^{2}\right)^{2} \gamma_{1}^{2}\left[3\left(2 b-b m^{2}+\nu\right) \beta_{1}+a\left(-2+m^{2}\right) \gamma_{1}\right]}}, \\
& q_{7}(x, t)=-\frac{\mathrm{i} \sqrt{6} \sqrt{1-m^{2}}\left(-1+2 m^{2}\right) \sqrt{\nu} \mathrm{nc}(x+\nu t) \gamma_{1}^{3 / 2}}{\sqrt{\left[b\left(-1+2 m^{2}\right)+\nu\right]^{3} \alpha_{1}+\left(1-2 m^{2}\right)^{2} \gamma_{1}^{2}\left[3\left(-b+2 b m^{2}+\nu\right) \beta_{1}+a\left(1-2 m^{2}\right) \gamma_{1}\right]}}, \\
& r_{7}(x, t)=-\frac{\mathrm{i} \sqrt{6} \sqrt{1-m^{2}} \sqrt{\nu}\left[b\left(-1+2 m^{2}\right)+\nu\right] \mathrm{nc}(x+\nu t) \sqrt{\gamma_{1}}}{\sqrt{\left[b\left(-1+2 m^{2}\right)+\nu\right]^{3} \alpha_{1}+\left(1-2 m^{2}\right)^{2} \gamma_{1}^{2}\left[3\left(-b+2 b m^{2}+\nu\right) \beta_{1}+a\left(1-2 m^{2}\right) \gamma_{1}\right]}}, \\
& q_{8}(x, t)=\frac{\sqrt{6} \sqrt{m^{2}-1}\left(2-m^{2}\right) \sqrt{\nu} \operatorname{nd}(x+\nu t) \gamma_{1}^{3 / 2}}{\sqrt{\left[b\left(-2+m^{2}\right)-\nu\right]^{3} \alpha_{1}-\left(-2+m^{2}\right)^{2} \gamma_{1}^{2}\left[3\left(2 b-b m^{2}+\nu\right) \beta_{1}+a\left(-2+m^{2}\right) \gamma_{1}\right]}}, \\
& r_{8}(x, t)=-\frac{\sqrt{6} \sqrt{m^{2}-1} \sqrt{\nu}\left[b\left(2-m^{2}\right)+\nu\right] \operatorname{nd}(x+\nu t) \sqrt{\gamma_{1}}}{\sqrt{\left[b\left(-2+m^{2}\right)-\nu\right]^{3} \alpha_{1}-\left(-2+m^{2}\right)^{2} \gamma_{1}^{2}\left[3\left(2 b-b m^{2}+\nu\right) \beta_{1}+a\left(-2+m^{2}\right) \gamma_{1}\right]}}, \\
& q_{9}(x, t)=-\frac{\sqrt{6} \sqrt{1-m^{2}}\left(2-m^{2}\right) \sqrt{\nu} \operatorname{sc}(x+\nu t) \gamma_{1}^{3 / 2}}{\sqrt{\left[b\left(-2+m^{2}\right)-\nu\right]^{3} \alpha_{1}-\left(-2+m^{2}\right)^{2} \gamma_{1}^{2}\left[3\left(2 b-b m^{2}+\nu\right) \beta_{1}+a\left(-2+m^{2}\right) \gamma_{1}\right]}}, \\
& r_{9}(x, t)=-\frac{\sqrt{6} \sqrt{1-m^{2}} \sqrt{\nu}\left[b\left(2-m^{2}\right)+\nu\right] \operatorname{sc}(x+\nu t) \sqrt{\gamma_{1}}}{\sqrt{\left[b\left(-2+m^{2}\right)-\nu\right]^{3} \alpha_{1}-\left(-2+m^{2}\right)^{2} \gamma_{1}^{2}\left[3\left(2 b-b m^{2}+\nu\right) \beta_{1}+a\left(-2+m^{2}\right) \gamma_{1}\right]}}, \\
& q_{10}(x, t)=-\frac{\mathrm{i} \sqrt{6} \sqrt{m^{2}\left(1+m^{2}\right)}\left(-1+2 m^{2}\right) \sqrt{\nu} \operatorname{sd}(x+\nu t) \gamma_{1}^{3 / 2}}{\sqrt{\left[b\left(-1+2 m^{2}\right)+\nu\right]^{3} \alpha_{1}+\left(1-2 m^{2}\right)^{2} \gamma_{1}^{2}\left[3\left(-b+2 b m^{2}+\nu\right) \beta_{1}+a\left(1-2 m^{2}\right) \gamma_{1}\right]}}, \\
& r_{10}(x, t)=-\frac{\mathrm{i} \sqrt{6} \sqrt{m^{2}\left(1+m^{2}\right)} \sqrt{\nu}\left[b\left(-1+2 m^{2}\right)+\nu\right] \operatorname{sd}(x+\nu t) \sqrt{\gamma_{1}}}{\sqrt{\left[b\left(-1+2 m^{2}\right)+\nu\right]^{3} \alpha_{1}+\left(1-2 m^{2}\right)^{2} \gamma_{1}^{2}\left[3\left(-b+2 b m^{2}+\nu\right) \beta_{1}+a\left(1-2 m^{2}\right) \gamma_{1}\right]}}, \\
& q_{11}(x, t)=-\frac{\sqrt{6}\left(2-m^{2}\right) \sqrt{\nu} \operatorname{cs}(x+\nu t) \gamma_{1}^{3 / 2}}{\sqrt{\left[b\left(-2+m^{2}\right)-\nu\right]^{3} \alpha_{1}-\left(-2+m^{2}\right)^{2} \gamma_{1}^{2}\left[3\left(2 b-b m^{2}+\nu\right) \beta_{1}+a\left(-2+m^{2}\right) \gamma_{1}\right]}}, \\
& r_{11}(x, t)=-\frac{\sqrt{6} \sqrt{\nu}\left[b\left(2-m^{2}\right)+\nu\right] \operatorname{cs}(x+\nu t) \sqrt{\gamma_{1}}}{\sqrt{\left[b\left(-2+m^{2}\right)-\nu\right]^{3} \alpha_{1}-\left(-2+m^{2}\right)^{2} \gamma_{1}^{2}\left[3\left(2 b-b m^{2}+\nu\right) \beta_{1}+a\left(-2+m^{2}\right) \gamma_{1}\right]}}, \\
& q_{12}(x, t)=-\frac{\sqrt{6}\left(-1+2 m^{2}\right) \sqrt{\nu} \mathrm{ds}(x+\nu t) \gamma_{1}^{3 / 2}}{\sqrt{\left[b\left(1-2 m^{2}\right)+\nu\right]^{3} \alpha_{1}-\left(1-2 m^{2}\right)^{2} \gamma_{1}^{2}\left[3\left(-b+2 b m^{2}+\nu\right) \beta_{1}+a\left(1-2 m^{2}\right) \gamma_{1}\right]}}, \\
& r_{12}(x, t)=-\frac{\sqrt{6} \sqrt{\nu}\left[b\left(-1+2 m^{2}\right)+\nu\right] \operatorname{ds}(x+\nu t) \sqrt{\gamma_{1}}}{\sqrt{\left[b\left(1-2 m^{2}\right)+\nu\right]^{3} \alpha_{1}-\left(1-2 m^{2}\right)^{2} \gamma_{1}^{2}\left[3\left(-b+2 b m^{2}+\nu\right) \beta_{1}+a\left(1-2 m^{2}\right) \gamma_{1}\right]}}, \\
& q_{13}(x, t)=-\frac{\mathrm{i} \sqrt{6} \sqrt{0.25} l \sqrt{\nu}[\operatorname{cs}(x+\nu t)+\mathrm{ns}(x+\nu t)] \gamma_{1}^{3 / 2}}{\sqrt{3 b l \nu^{2} \alpha_{1}+\nu^{3} \alpha_{1}+l^{2}\left(3 b^{2} \nu \alpha_{1}+3 \nu \beta_{1} \gamma_{1}^{2}\right)+l^{3}\left(b^{3} \alpha_{1}+3 b \beta_{1} \gamma_{1}^{2}-a \gamma_{1}^{3}\right)}},
\end{aligned}
$$




$$
r_{13}(x, t)=-\frac{\mathrm{i} \sqrt{6} \sqrt{0.25} \sqrt{\nu}(b l+\nu)[\operatorname{cs}(x+\nu t)+\operatorname{ns}(x+\nu t)] \sqrt{\gamma_{1}}}{\sqrt{3 b l \nu^{2} \alpha_{1}+\nu^{3} \alpha_{1}+l^{2}\left(3 b^{2} \nu \alpha_{1}+3 \nu \beta_{1} \gamma_{1}^{2}\right)+l^{3}\left(b^{3} \alpha_{1}+3 b \beta_{1} \gamma_{1}^{2}-a \gamma_{1}^{3}\right)}},
$$

where $l=\left(0.5-m^{2}\right)$,

$$
\begin{aligned}
& q_{14}(x, t)=-\frac{\mathrm{i} \sqrt{6} \sqrt{0.5-0.5 m^{2}}\left(0.5+0.5 m^{2}\right) \sqrt{\nu}[\operatorname{nc}(x+\nu t)+\operatorname{sc}(x+\nu t)] \gamma_{1}^{3 / 2}}{\sqrt{3 b s \nu^{2} \alpha_{1}+\nu^{3} \alpha_{1}+s^{2}\left(3 b^{2} \nu \alpha_{1}+3 \nu \beta_{1} \gamma_{1}^{2}\right)+s^{3}\left(b^{3} \alpha_{1}+3 b \beta_{1} \gamma_{1}^{2}-a \gamma_{1}^{3}\right)}}, \\
& r_{14}(x, t)=-\frac{\mathrm{i} \sqrt{6} \sqrt{0.5-0.5 m^{2}} \sqrt{\nu}(b s+\nu)[\operatorname{nc}(x+\nu t)+\operatorname{sc}(x+\nu t)] \sqrt{\gamma_{1}}}{\sqrt{3 b s \nu^{2} \alpha_{1}+\nu^{3} \alpha_{1}+s^{2}\left(3 b^{2} \nu \alpha_{1}+3 \nu \beta_{1} \gamma_{1}^{2}\right)+s^{3}\left(b^{3} \alpha_{1}+3 b \beta_{1} \gamma_{1}^{2}-a \gamma_{1}^{3}\right)}},
\end{aligned}
$$

where $s=\left(0.5+0.5 m^{2}\right)$,

$$
\begin{aligned}
& q_{15}(x, t)=\frac{\sqrt{6} \sqrt{0.25 m^{2}} h \sqrt{\nu}[\operatorname{ds}(x+\nu t)+\operatorname{ns}(x+\nu t)] \gamma_{1}^{3 / 2}}{\sqrt{3 b h \nu^{2} \alpha_{1}-\nu^{3} \alpha_{1}+h^{2}\left(3 b^{2} \nu \alpha_{1}+3 \nu \beta_{1} \gamma_{1}^{2}\right)+h^{3}\left(b^{3} \alpha_{1}+3 b \beta_{1} \gamma_{1}^{2}-a \gamma_{1}^{3}\right)}}, \\
& r_{15}(x, t)=-\frac{\sqrt{6} \sqrt{0.25 m^{2}} \sqrt{\nu}(-b h+\nu)[\operatorname{ds}(x+\nu t)+\operatorname{ns}(x+\nu t)] \sqrt{\gamma_{1}}}{\sqrt{3 b h \nu^{2} \alpha_{1}-\nu^{3} \alpha_{1}+h^{2}\left(3 b^{2} \nu \alpha_{1}+3 \nu \beta_{1} \gamma_{1}^{2}\right)+h^{3}\left(b^{3} \alpha_{1}+3 b \beta_{1} \gamma_{1}^{2}-a \gamma_{1}^{3}\right)}},
\end{aligned}
$$

where $h=\left(1-0.5 m^{2}\right)$,

$$
\begin{aligned}
& q_{16}(x, t)=-\frac{\sqrt{6} \sqrt{0.25 m^{2}} h \sqrt{\nu}[\mathrm{i} \operatorname{cs}(x+\nu t)+\operatorname{sn}(x+\nu t)] \gamma_{1}^{3 / 2}}{\sqrt{3 b h \nu^{2} \alpha_{1}-\nu^{3} \alpha_{1}+h^{2}\left(3 b^{2} \nu \alpha_{1}+3 \nu \beta_{1} \gamma_{1}^{2}\right)+h^{3}\left(b^{3} \alpha_{1}+3 b \beta_{1} \gamma_{1}^{2}-a \gamma_{1}^{3}\right)}}, \\
& r_{16}(x, t)=-\frac{\mathrm{i} \sqrt{6} \sqrt{0.25 m^{2}} \sqrt{\nu}(-b h+\nu)[\mathrm{i} \operatorname{cs}(x+\nu t)+\operatorname{sn}(x+\nu t)] \sqrt{\gamma_{1}}}{\sqrt{3 b h \nu^{2} \alpha_{1}-\nu^{3} \alpha_{1}+h^{2}\left(3 b^{2} \nu \alpha_{1}+3 \nu \beta_{1} \gamma_{1}^{2}\right)+h^{3}\left(b^{3} \alpha_{1}+3 b \beta_{1} \gamma_{1}^{2}-a \gamma_{1}^{3}\right)}} .
\end{aligned}
$$

\section{Conclusions}

This paper derived the topological solitons or shock wave solutions to the GG model that is studied with $\mathrm{cu}-$ bic as well as power law nonlinearity. The Lie symmetry analysis is applied to extract the conserved density. Furthermore, the conserved quantity is derived from this density by using the 1-soliton solution. The FE method was then subsequently applied to extract several other solutions of the governing equation. These include singular periodic solutions, singular solitons, cnoidal waves, snoidal waves and several other doubly periodic functions. These plethora of solutions are very useful in conducting further research in the area of oceanography where GG model is studied.

In future, several other issues will be addressed. These are the integration of the equation using Lie symmetry, $G^{\prime} / G$-expansion method, exp-function approach and many more. These will lead to a few more interesting solutions and of course several of those solutions will overlap with the solutions that are listed in this paper. Moreover, GG model with time-dependent and/or stochastic coefficients are going to be taken into consideration. The results of those research will be available in future. This is just a tip of the iceberg.

\section{References}

[1] A.H. Bhrawy, M.A. Abdelkawy, A. Biswas, Commun Nonlin. Sci. Num. Simul. 18, 915 (2013).

[2] A.H. Bhrawy, M.A. Abdelkawy, S. Kumar, S. Johnson, A. Biswas, Indian J. Phys. 87, 455 (2013).

[3] A.H. Bhrawy, M.A. Abdelkawy, S. Kumar, A. Biswas, Romanian J. Phys. 58, 729 (2013).
[4] E. Bisognin, V. Bisognin, M. Sepulveda, O. Vera, J. Comput. Appl. Math. 220, 290 (2008).

[5] A. Biswas, M.S. Ismail, Appl. Math. Comput. 216, $3662(2010)$.

[6] A. Biswas, E.V. Krishnan, P. Suarez, A.H. Kara, S. Kumar, Indian J. Phys. 87, 169 (2013).

[7] U. Göktas, W. Hereman, Physica D 123, 425 (1998).

[8] Y. Guo, Y. Wang, Appl. Math. Comput. 217, 8080 (2011).

[9] A.J.M. Jawad, S. Johnson, A. Yildirim, S. Kumar, A. Biswas, Indian J. Phys. 87, 281 (2013).

[10] A.H. Kara, J. Nonlin. Math. Phys. 16, 149 (2009).

[11] Y.S. Kivshar, B.A. Malomed, Wave Motion 11, 261 (1989).

[12] Y. Kivshar, B.A. Malomed, Rev. Mod. Phys. 61, 763 (1989).

[13] H. Triki, M.S. Ismail, Appl. Math. Comput. 217, 1540 (2010).

[14] A.M. Wazwaz, Appl. Math. Comput. 184, 1002 (2007).

[15] H. Zhang, Commun. Nonlin. Sci. Num. Simul. 12, 1120 (2009). 